\title{
Co-effect of Serum Galectin-3 and High Density Lipoprotein- Cholesterol on the Prognosis of Acute Ischemic Stroke
}

Tan Xu; Nimei Zeng; Aili Wang; Yonghong Zhang

Department of Epidemiology, School of Public Health, Medical College of Soochow University, Suzhou, China

Objective: The association of combined galectin-3 and HDL-C with prognosis of acute ischemic stroke remains unknown. This study aimed to evaluate the co-effect of galectin-3 and HDL-C on death and vascular events within one year after ischemic stroke.

Methods: Based on China Antihypertensive Trial in Acute Ischemic Stroke (CATIS), a prospective study was conducted among 2819 patients with acute ischemic stroke. The study outcome was a combination of death and vascular events, within one year after ischemic stroke. The secondary outcome was separately those of recurrent stroke, vascular events and death.

Results: During the one-year follow-up period, a total of 238 patients experienced primary outcome. The multivariate adjusted hazard ratios $(95 \%$ confidence intervals) of primary outcome, recurrent stroke and vascular events were 1.55(1.06-2.25), 1.82(1.10-3.02) and 1.87(1.21-2.90), respectively, in patients with both high galectin-3 and low HDL-C compared to those with both low galectin-3 and high HDL-C. The addition of galectin-3 and HDL-C to conventional factors significantly improved predictive value. Net reclassification index was $15.7 \%$ for primary outcome, $18.3 \%$ for recurrent stroke and $20.5 \%$ for vascular events.

Conclusion: Combination of high galectin-3 and low HDL-C was associated with of primary outcome, recurrent stroke and vascular events within one year after ischemic stroke, suggesting that the combination of galectin-3 and HDL-C should be used to identify the individuals at risk of poor prognosis after ischemic stroke.

Key words: galectin-3, high density lipoproteincholesterol, prognosis, ischemic stroke 